\title{
Broadband metasurfaces enabling arbitrarily large delay-bandwidth products
}

\author{
Vincent Ginis, ${ }^{1, a)}$ Philippe Tassin, ${ }^{2}$ Thomas Koschny, ${ }^{3}$ and Costas M. Soukoulis ${ }^{3,4}$ \\ ${ }^{1}$ Applied Physics Research Group (APHY), Vrije Universiteit Brussel, Pleinlaan 2, B-1050 Brussel, Belgium \\ ${ }^{2}$ Department of Applied Physics, Chalmers University, SE-412 96 Göteborg, Sweden \\ ${ }^{3}$ Ames Laboratory - U.S. DOE and Department of Physics and Astronomy, Iowa State University, Ames, \\ Iowa 50011, USA \\ ${ }^{4}$ Institute of Electronic Structure and Lasers (IESL), FORTH, GR-71110 Heraklion, Crete, Greece
}

(Received 6 November 2015; accepted 5 January 2016; published online 19 January 2016)

\begin{abstract}
Metasurfaces allow for advanced manipulation of optical signals by imposing phase discontinuities across flat interfaces. Unfortunately, these phase shifts remain restricted to values between 0 and $2 \pi$, limiting the delay-bandwidth product of such sheets. Here, we develop an analytical tool to design metasurfaces that mimic three-dimensional materials of arbitrary thickness. In this way, we demonstrate how large phase discontinuities can be realized by combining several subwavelength Lorentzian resonances in the unit cell of the surface. Our methods open up the temporal response of metasurfaces and may lead to the construction of metasurfaces with a plethora of new optical functions. (C) 2016 AIP Publishing LLC. [http://dx.doi.org/10.1063/1.4939979]
\end{abstract}

A sheet metamaterial or metasurface is a two-dimensional patterned structure whose electromagnetic properties are determined by subwavelength resonant elements. These singlelayer extensions of bulky metamaterials ${ }^{1-5}$ have revolutionized the manipulation of optical signals as they allow for controlling light in a deep subwavelength regime. Starting from the observation that the laws of reflection and refraction can be generalized to include phase jumps at the interface, ${ }^{6}$ a new line of research has emerged around artificial surfaces that reflect and refract light in unconventional ways. ${ }^{7-9}$ This approach has led to a class of surfaces that introduce nontrivial phase discontinuities and may replace many conventional bulky devices, whose functionality relies on the specific phase accumulation of light. Indeed, metasurfaces with spatially varying phase discontinuities allow for ultra-thin analogues of traditional lenses and axicons, ${ }^{10}$ focusing mirrors, ${ }^{11}$ compact polarimeters, ${ }^{12}$ and anomalous reflection and refraction. ${ }^{13,14}$ In addition, several groups have demonstrated perfect absorption using metasurfaces that exhibit a nontrivial phase shift in reflection. ${ }^{15-17}$ It is also possible to manipulate the polarization of light using surfaces with different spectral response along two orthogonal directions. ${ }^{18}$ In this way, anisotropic metasurfaces can implement wave plates, vortex plates, chromatic polarizers, and surface plasmon polariton couplers. ${ }^{19-23}$ Two generalizations have considerably increased the efficiency of metasurfaces. Electromagnetic metasurfaces that exhibit both an electrical and a magnetic phase discontinuity, ${ }^{24}$ and meta-transmit-arrays that consist of a sequence of metasurfaces ${ }^{25}$ may facilitate highly efficient, reflectionless manipulation of the polarization, phase and amplitude of incoming pulses. So far, the overall phase shift that is imposed by these metasurfaces remains bounded between 0 and $\pi$ for electric response sheets and between 0 and $2 \pi$ for electromagnetic sheets. In addition, their bandwidth is normally restricted due to their explicit resonant operation.

In this letter, we demonstrate that it is possible to expand the range of phase discontinuities to much larger values, opening up the temporal response of metasurfaces, and we

\footnotetext{
${ }^{\text {a) }}$ Author to whom correspondence should be addressed. Email: vincent.ginis@ vub.ac.be
}

discuss the application of such metasurfaces for broadband pulse delay. In addition, we demonstrate that it is possible to achieve a metamaterial in an a priori determined frequency band, i.e., the frequency band of operation is not restricted by the resonant properties associated with the desired response function. We start our analysis from the observation that there is a one-to-one correspondence between the electric $\left(\sigma_{\mathrm{se}}\right)$ and magnetic sheet $\left(\sigma_{\mathrm{sm}}\right)$ conductivities of metasurfaces and their scattering amplitudes ${ }^{24,26}$

$$
\begin{gathered}
\sigma_{\mathrm{se}}\left(\omega, k_{\perp}\right)=\frac{2}{\xi\left(\omega, k_{\perp}\right)} \frac{1-R\left(\omega, k_{\perp}\right)-T\left(\omega, k_{\perp}\right)}{1+R\left(\omega, k_{\perp}\right)+T\left(\omega, k_{\perp}\right)}, \\
\sigma_{\mathrm{sm}}\left(\omega, k_{\perp}\right)=2 \xi\left(\omega, k_{\perp}\right) \frac{1+R\left(\omega, k_{\perp}\right)-T\left(\omega, k_{\perp}\right)}{1-R\left(\omega, k_{\perp}\right)+T\left(\omega, k_{\perp}\right)},
\end{gathered}
$$

in which $R\left(\omega, k_{\perp}\right)$ and $T\left(\omega, k_{\perp}\right)$ are the reflection and transmission amplitudes, respectively. The wave impedance $\xi\left(\omega, k_{\perp}\right)$ equals $\omega \mu_{0} / k_{\perp}$. For normal incident waves, this simplifies to the free-space impedance $\xi \approx 377 \Omega$. To obtain the sheet conductivities that impose an arbitrarily large phase shift on a broadband incoming pulse, we can insert the scattering parameters of a dispersionless matched slab of thickness $d$ and refractive index $n$, i.e., $R(\omega)=0$ and $T(\omega)=A \exp [\mathrm{i} \omega(n-1) d / c]$, where the parameter $A$ accounts for a possible amplitude decrease in the transmitted field $(A<1)$ and where the reference planes of the incoming (outgoing) waves coincide with the leftmost (rightmost) boundary of the slab. Writing the corresponding fraction as a series expansion, we arrive at

$$
\frac{\xi \sigma_{\mathrm{se}}(\omega)}{2}=\frac{\sigma_{\mathrm{sm}}(\omega)}{2 \xi}=1+2 \sum_{m=1}^{\infty}(-A)^{m} \exp \left(\mathrm{i} \omega m t_{0}\right),
$$

where $t_{0}=(n-1) d / c$ is the difference between the phase delay time through the matched slab and the phase delay time through a vacuum region of the same thickness. These conductivities are causal periodic functions of $\omega$ with periodicity $\pi / t_{0}$. In Fig. 1 , we plot the first two periods of this function. The resonances of the conductivities occur at those frequencies for which the optical path difference between 


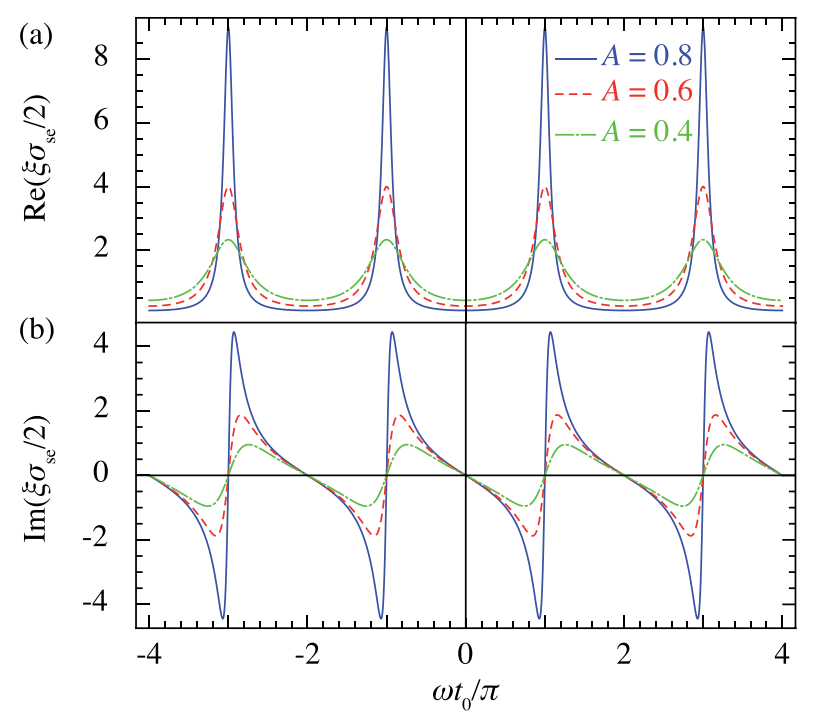

FIG. 1. The first two periods of the real and the imaginary parts of the surface conductivities as a function of frequency for several values of the transmission amplitude $A$.

the slab and vacuum of the same thickness equals an odd multiple of half the free-space wavelength. These are the frequencies for which a wave propagating through vacuum and a wave propagating through the slab are in anti-phase. The zeros of the susceptance $\left[\operatorname{Im}\left(\sigma_{\mathrm{se}}\right)\right]$, on the other hand, correspond to the frequencies for which the aforementioned waves are in phase with each other and for which the sheet should only generate an amplitude decrease, in correspondence with the nonzero real part of the conductivities.

The physics behind the time delay of this metasurface can be understood by evaluating the time-domain representation of the conductivities. Therefore, we calculate the inverse Fourier transform of Eq. (1), which yields

$$
\frac{\xi \sigma_{\mathrm{se}}(t)}{2}=\frac{\sigma_{\mathrm{sm}}(t)}{2 \xi}=\delta(t)+2 \sum_{m=1}^{\infty}(-A)^{m} \delta\left(t-m t_{0}\right)
$$

The electric and magnetic currents flowing on the metasurface are given by the convolution of these conductivity kernels with the local electromagnetic field. In Fig. 2(a), we show that the impulse response of the currents flowing on the surface is a Dirac pulse train whose amplitude is decreasing with a factor $A$ at each subsequent pulse. It can be shown that for an arbitrary incident field $E_{\text {in }}(t), H_{\text {in }}(t)$ the electric and magnetic sheet currents are given by (see the supplementary material) ${ }^{27}$

$$
\begin{gathered}
\xi j_{\mathrm{se}}(t)=E_{\text {in }}(t)-A E_{\text {in }}\left(t-t_{0}\right), \\
\xi^{-1} j_{\text {sm }}(t)=H_{\text {in }}(t)-A H_{\text {in }}\left(t-t_{0}\right) .
\end{gathered}
$$

As a result, the outgoing field is a replica of the incident one modulated with the appropriate amplitude $A$ and phase shift $\exp \left(\mathrm{i} \omega t_{0}\right)$

$$
\begin{aligned}
& E_{\text {out }}(t)=A E_{\text {in }}\left(t-t_{0}\right), \\
& H_{\text {out }}(t)=A H_{\text {in }}\left(t-t_{0}\right),
\end{aligned}
$$

as shown in Fig. 2(b).

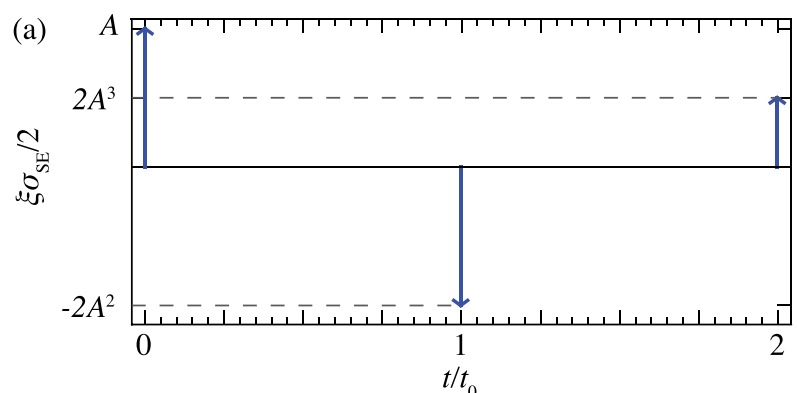

(b)

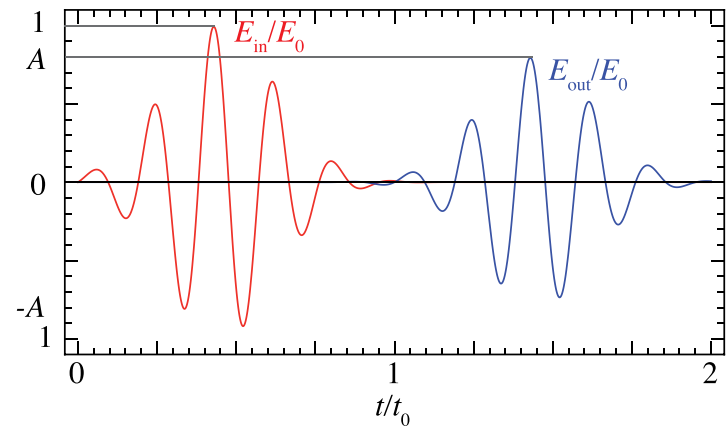

FIG. 2. (a) The time-domain representation of the ideal surface conductivities is a Dirac pulse train whose amplitude decays with a factor $A$ at each consecutive pulse. The surface currents on the sheet are defined by the convolution of the local field on the surface with these conductivities. (b) For an arbitrary incident pulse (red), the outgoing pulse is an exact replica of the incident one with a shifted phase and amplitude (blue).

The highly singular nature of the impulse response of the conductivities is a result of the fact that this sheet mimics a nondispersive slab that adds a propagation phase to the incident waves of all frequencies. However, in any physical realization of this surface, there exists an upper limit on the frequency for which this assumption remains valid. Furthermore, only specific types of resonant behavior are available in nature. Therefore, we investigated whether the spectrum defined in Eq. (1) can be approximated by a sum of Lorentzian resonances, which can be easily obtained with subwavelength meta-atoms. Using a partial fraction decomposition at the poles $p_{m}$ of Eq. (1), we can show that the desired spectrum of conductivities can be written as the conductivities corresponding to a sum of Lorentzians in the susceptibility $^{27}$

$$
\sum_{m=0}^{+\infty} \frac{-4 \mathrm{i} \omega / t_{0}}{\left|p_{m}\right|^{2}+2 \mathrm{iIm}\left(p_{m}\right) \omega-\omega^{2}},
$$

with an extra residual term

$$
\sum_{m=0}^{+\infty} \frac{-4 \operatorname{Im}\left(p_{m}\right) / t_{0}}{\left|p_{m}\right|^{2}+2 \operatorname{iIm}\left(p_{m}\right) \omega-\omega^{2}},
$$

where the $m$ th pole of the transmission function is located at $p_{m}=(2 m+1) \pi / t_{0}-\mathrm{i} \log (1 / a) / t_{0}$. The residual term, whose relative magnitude rapidly decreases at nonzero frequencies, accounts for the fact that the real part of Eq. (1) has a nonzero contribution at DC. Apart from the DC discrepancy, our technique also works perfectly in the non-ideal case of lossy oscillators. In other words, the nonzero background in the real part of the conductivities in between the 
resonances can be fitted arbitrarily good using a specific sum of Lorentzians oscillators.

In Fig. 3, we evaluate the transmission through a metasurface approximating the ideal sheet conductivities defined in Eq. (1) by a finite sum of Lorentzians, corresponding to the first $N$ terms of Eq. (5). The impulse response of these realistic conductivities is no longer a singular Dirac train, but instead an oscillatory function whose extrema decrease as a function of time, as shown in Fig. 3(a). Consequently, there exists an upper limit on the bandwidth of operation for any given time delay. More generally, the delay-bandwidth product depends on the number of Lorentzian resonances that is implemented in the surface. By truncating the series of resonances, the
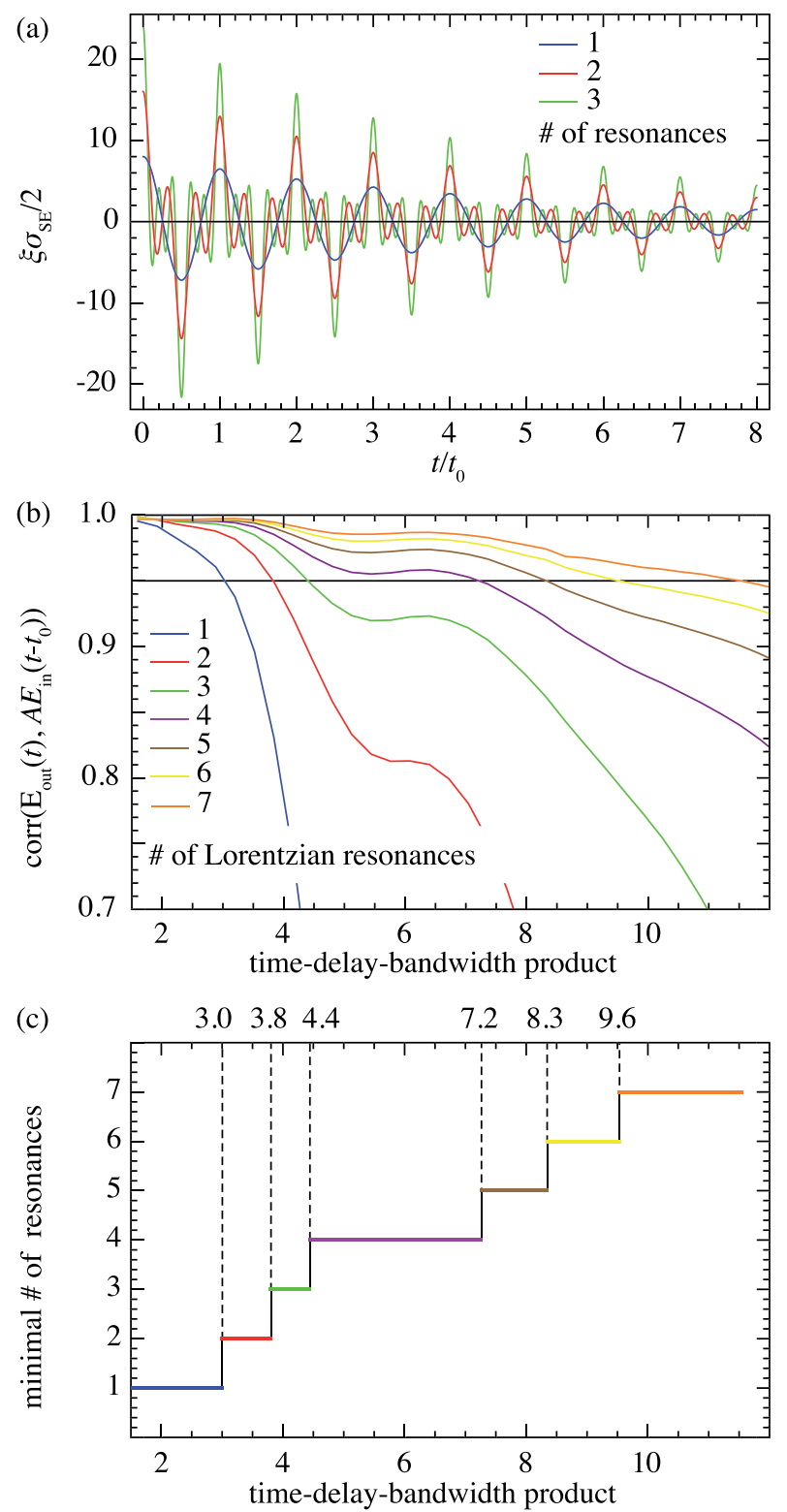

FIG. 3. (a) The impulse response of the sheet conductivities $(A=0.9)$ of the approximated sheets, incorporating 1, 2, or 3 Lorentzian resonances. (b) The numerically calculated cross-correlation between the pulse transmitted through the approximated sheet and the ideal amplitude-shifted $(A=0.9)$ and phase-shifted pulse as a function of the number of Lorentzian resonances. This incident wave is a broadband Gaussian pulse (pulse width $\Delta t=5 / \omega_{0}$ ). (c) The extracted minimal number of Lorentzians versus delaybandwidth product requiring a 95\% cross-correlation level between the pulses transmitted through the ideal and the realistic sheets. shape of the delayed pulse will not be preserved when the spectral content of the incident pulse is too broad. To investigate this effect, we numerically simulate the transmission of a Gaussian pulse through the metasurface and compare this delayed pulse with the transmitted pulse of the ideal system, given in Eqs. (3) and (4), as a function of the resulting time delay $t_{0}$. The pulse distortion is quantified by the crosscorrelation between both pulses. In Fig. 3(b), we plot this cross-correlation for several surfaces implementing a different number of Lorentzian resonances. As expected, the crosscorrelation decreases as the delay-bandwidth product increases and the gradient of this curve highly depends on the number of resonances. From this figure, we can extract the minimal number of resonances that must be implemented for a given delay-bandwidth product by imposing a crosscorrelation of at least $95 \%$ between the transmitted pulses through the ideal and the approximated surface. This relation, shown in Fig. 3(c), demonstrates that there is no fundamental limit on the delay-bandwidth product of a matched surface. Our approach thus allows to design a metasurface with an arbitrarily large delay-bandwidth product. In contrast with most other metamaterials, we can also choose the bandwidth at the design stage. The bandwidth is not fundamentally limited by the resonant response. A surface with 4 resonances, e.g., outperforms traditional metamaterial slow-light implementations by an order of magnitude and approaches the delay-bandwidth products of atomic vapors. ${ }^{28-33}$
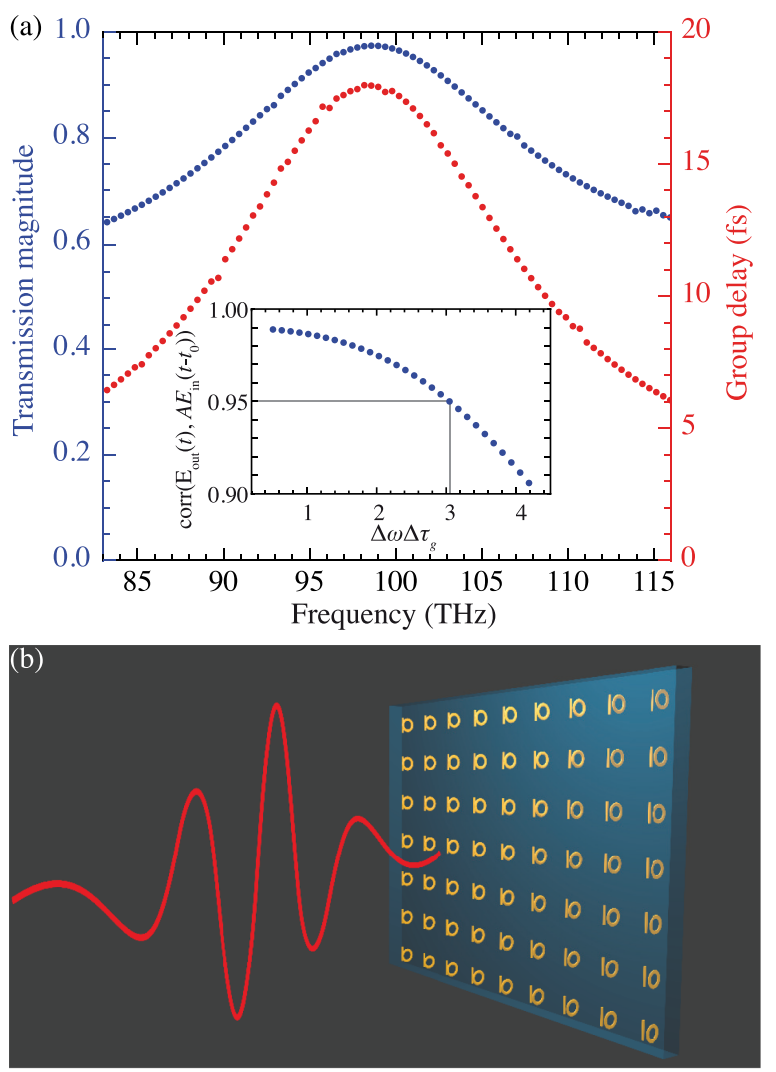

FIG. 4. (a) The numerically retrieved transmission parameters of a metasurface in which the macroscopic conductivities of one Lorentzian pair are implemented with electric and magnetic dipoles with Lorentzian response (setup shown in (b)). The inset in (a) shows the correlation between incident and transmitted pulse versus the delay-bandwidth product. 
Subsequently, we have verified these results using finite element numerical simulations. ${ }^{27} \mathrm{We}$ simulated an implementation of the desired conductivities approximated by the first Lorentzian in the electric and in the magnetic response. The resonance is achieved through electric and magnetic point dipoles with a microscopic Lorentzian response. This response can be implemented with lumped elements up to microwave frequencies, with standard metallic metamaterials up to infrared frequencies ${ }^{1-4}$ and with nano circuit elements at optical frequencies. ${ }^{34,35}$ The results in Fig. 4 show the numerically retrieved values of the magnitude of the transmittance and group delay of this metasurface. We extracted the timedelay-bandwidth product of these parameters by calculating the cross-correlation between the incident and transmitted pulse as a function of the incident pulse bandwidth and found agreement $\left(\Delta \omega \Delta \tau_{g}=3.05 \pm 0.06\right)$ with the analytical findings from Fig. $3\left(\Delta \omega \Delta \tau_{g}=3.04\right)$. The design procedure decouples the losses, determined by the transmission amplitude $A$, from the delay-bandwidth product, determined by the number of Lorentzian oscillators and their periodicity $\pi / t_{0}$ in the frequency domain.

In conclusion, we have demonstrated that thin-sheet metamaterials allow for broadband phase manipulation. The phase shift experienced by an incoming pulse passing through the sheet can become arbitrarily large. Furthermore, we have shown that the material response to achieve this functionality can be approximated by a sum of Lorentzian resonances that can be implemented by meta-atoms. We studied the dependence of the delay-bandwidth product versus the number of implemented resonances and found that our approach dramatically extends the reach of metasurfaces for the manipulation of electromagnetic signals, introducing electromagnetic sheets for broadband phase manipulation. The understanding of metamaterials as a function of multiple Lorentzian resonators may also facilitate the further development of nonlinear, tunable, and active metamaterials. ${ }^{36-38}$ Our methods can be further used to achieve other optical functions with subwavelength metamaterial structures based on a finite number of superimposed Lorentzian meta-atoms.

Work at the VUB (simulations) was supported by BelSPO (Grant No. IAP P7-35 photonics@be) and the Research Foundation-Flanders. Work at Ames Lab (theory) was supported by the US Department of Energy, Office of Basic Energy Science, Division of Materials Sciences and Engineering under Contract No. DE-AC02- 07CH11358). Work at FORTH (modeling) was supported by the European Research Council under ERC Advanced Grant No. 320081 (PHOTOMETA). V.G. acknowledges support as a postdoctoral Fellow of the Research Foundation-Flanders (FWO- Vlaanderen).
${ }^{1}$ D. R. Smith, J. B. Pendry, and M. C. K. Wiltshire, Science 305, 788 (2004).

${ }^{2}$ R. Engheta and R. W. Ziolkowski, Metamaterials, Physics and Engineering Explorations (Wiley-IEEE Press, New York, 2006).

${ }^{3}$ Y. Liu and X. Zhang, Chem. Soc. Rev. 40, 2494 (2011).

${ }^{4}$ C. M. Soukoulis and M. Wegener, Nat. Photon. 5, 523 (2011).

${ }^{5}$ N. I. Zheludev and Y. S. Kivshar, Nat. Mater. 11, 917 (2012).

${ }^{6}$ N. Yu, P. Genevet, M. A. Kats, F. Aieta, J.-P. Tetienne, F. Capasso, and Z. Gaburro, Science 334, 333 (2011).

${ }^{7}$ C. Holloway, E. F. Kuester, J. Gordon, J. O'Hara, J. Booth, and D. Smith, IEEE Antennas Propag. Mag. 54, 10 (2012).

${ }^{8}$ A. V. Kildishev, A. Boltasseva, and V. M. Shalaev, Science 339, 1232009 (2013).

${ }^{9}$ N. Yu, P. Genevet, F. Aieta, M. Kats, R. Blanchard, G. Aoust, J.-P. Tetienne, Z. Gaburro, and F. Capasso, IEEE J. Sel. Top. Quantum Electron. 19, 4700423 (2013).

${ }^{10}$ F. Aieta, P. Genevet, M. A. Kats, N. Yu, R. Blanchard, Z. Gaburro, and F. Capasso, Nano Lett. 12, 4932 (2012).

${ }^{11}$ A. Pors, M. G. Nielsen, R. L. Eriksen, and S. I. Bozhevolnyi, Nano Lett. 13, 829 (2013).

${ }^{12}$ J. B. Mueller, K. Leosson, and F. Capasso, Nano Lett. 14, 5524 (2014).

${ }^{13}$ F. Aieta, P. Genevet, N. Yu, M. A. Kats, Z. Gaburro, and F. Capasso, Nano Lett. 12, 1702 (2012).

${ }^{14}$ L. Huang, X. Chen, H. Mühlenbernd, G. Li, B. Bai, Q. Tan, G. Jin, T. Zentgraf, and S. Zhang, Nano Lett. 12, 5750 (2012).

${ }^{15}$ M. A. Kats, D. Sharma, J. Lin, P. Genevet, R. Blanchard, Z. Yang, M. M. Qazilbash, D. Basov, S. Ramanathan, and F. Capasso, Appl. Phys. Lett. 101, 221101 (2012).

${ }^{16}$ H.-T. Chen, Opt. Express 20, 7165 (2012).

${ }^{17}$ C. Argyropoulos, K. Q. Le, N. Mattiucci, G. D'Aguanno, and A. Alù, Phys. Rev. B 87, 205112 (2013).

${ }^{18}$ Y. Zhao and A. Alù, Phys. Rev. B 84, 205428 (2011).

${ }^{19}$ E. H. Khoo, E. P. Li, and K. B. Crozier, Opt. Lett. 36, 2498 (2011).

${ }^{20}$ T. Ellenbogen, K. Seo, and K. B. Crozier, Nano Lett. 12, 1026 (2012).

${ }^{21}$ P. Genevet, J. Lin, M. A. Kats, and F. Capasso, Nat. Commun. 3, 1278 (2012).

${ }^{22}$ J. Lin, J. B. Mueller, Q. Wang, G. Yuan, N. Antoniou, X.-C. Yuan, and F. Capasso, Science 340, 331 (2013).

${ }^{23}$ L. Huang, X. Chen, B. Bai, Q. Tan, G. Jin, T. Zentgraf, and S. Zhang, Light Sci. Appl. 2, e70 (2013).

${ }^{24}$ C. Pfeiffer and A. Grbic, Phys. Rev. Lett. 110, 197401 (2013).

${ }^{25}$ F. Monticone, N. M. Estakhri, and A. Alù, Phys. Rev. Lett. 110, 203903 (2013).

${ }^{26} \mathrm{P}$. Tassin, T. Koschny, and C. M. Soukoulis, Physica B 407, 4062 (2012).

${ }^{27}$ See supplementary material at http://dx.doi.org/10.1063/1.4939979 for the analytical derivation of the broadband phase delay, the fitting of the target spectrum to a sum of Lorentzians, and the details of the finite elements numerical simulations.

${ }^{28}$ J. B. Khurgin, JOSA B 22, 1062 (2005).

${ }^{29}$ S. Zhang, D. A. Genov, Y. Wang, M. Liu, and X. Zhang, Phys. Rev. Lett. 101, 047401 (2008).

${ }^{30}$ N. Papasimakis, V. A. Fedotov, N. I. Zheludev, and S. L. Prosvirnin, Phys. Rev. Lett. 101, 253903 (2008).

${ }^{31}$ P. Tassin, L. Zhang, T. Koschny, E. Economou, and C. Soukoulis, Phys. Rev. Lett. 102, 053901 (2009).

${ }^{32}$ N. Liu, L. Langguth, T. Weiss, J. Kästel, M. Fleischhauer, T. Pfau, and H. Giessen, Nat. Mater. 8, 758 (2009).

${ }^{33}$ L. Verslegers, Z. Yu, Z. Ruan, P. B. Catrysse, and S. Fan, Phys. Rev. Lett. 108, 083902 (2012).

${ }^{34}$ A. Alu and N. Engheta, Phys. Rev. B 75, 024304 (2007).

${ }^{35}$ N. Engheta, Science 317, 1698 (2007).

${ }^{36}$ C. Kurter, P. Tassin, A. P. Zhuravel, L. Zhang, T. Koschny, A. V. Ustinov, C. M. Soukoulis, and S. M. Anlage, Appl. Phys. Lett. 100, 121906 (2012).

${ }^{37}$ I. V. Shadrivov, M. Lapine, and Y. S. Kivshar, Nonlinear, Tunable and Active Metamaterials (Springer, 2015).

${ }^{38}$ V. Ginis, P. Tassin, B. Craps, and I. Veretennicoff, Opt. Express 18, 5350 (2010). 